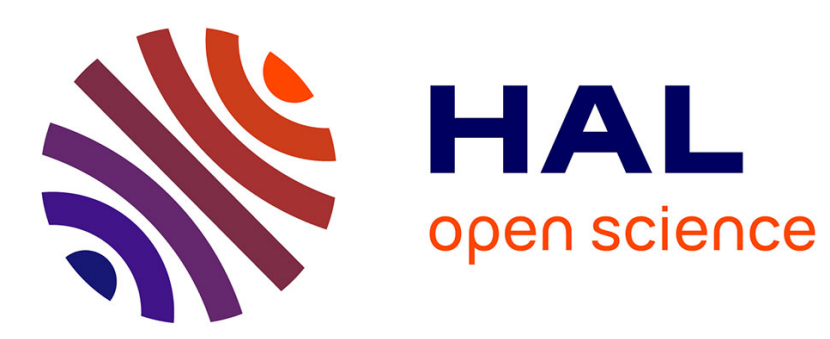

\title{
Transition ou consolidation du régime dominant : le métabolisme urbain en question
}

\author{
Sabine Barles, Jean-Baptiste Bahers
}

\section{To cite this version:}

Sabine Barles, Jean-Baptiste Bahers. Transition ou consolidation du régime dominant : le métabolisme urbain en question. Flux - Cahiers scientifiques internationaux Réseaux et territoires, 116-117 (2), pp.1, 2019, 10.3917/flux1.116.0001 . halshs-02462444

\section{HAL Id: halshs-02462444 https://shs.hal.science/halshs-02462444}

Submitted on 31 Jan 2020

HAL is a multi-disciplinary open access archive for the deposit and dissemination of scientific research documents, whether they are published or not. The documents may come from teaching and research institutions in France or abroad, or from public or private research centers.
L'archive ouverte pluridisciplinaire HAL, est destinée au dépôt et à la diffusion de documents scientifiques de niveau recherche, publiés ou non, émanant des établissements d'enseignement et de recherche français ou étrangers, des laboratoires publics ou privés. 


\section{« Transition ou consolidation du régime dominant : le métabolisme urbain en question »}

\section{Pour une approche interdisciplinaire du métabolisme urbain et de ses transformations}

1Si les politiques d'économie circulaire fleurissent en France et ailleurs, la connaissance du métabolisme urbain, entendu comme l'ensemble des flux d'énergie et de matière permettant le fonctionnement des villes, demeure aujourd'hui lacunaire. Les travaux en la matière ont connu un regain d'intérêt depuis quelques années (Weisz, Steinberger, 2010 ; Zhang, Yang, $\mathrm{Yu}, 2015)$, mais, bien que toujours plus nombreux, restent dispersés de par le monde, souvent concentrés sur les métropoles conçues comme la forme paroxystique et dominante de l'urbanisation planétaire (Ferrao, Fernández, 2013) - l'énormité des flux de matières et d'énergie qu'elles mettent en jeu ne laisse en effet pas d'être préoccupante (voir par exemple : Kennedy et alii, 2015). Ces travaux sont souvent ancrés dans une approche quantitative qui néglige le caractère multiscalaire du métabolisme urbain et plus généralement territorial. Ils peinent par ailleurs à envisager le métabolisme comme une coproduction socio-écologique et, lorsqu'ils le font, s'inscrivent la plupart du temps dans une visée prescriptive et normative placée sous le sceau de la transition, nouvel étendard de la modernisation écologique et du progrès, sans vraiment questionner les tenants et aboutissants de celle-ci.

2Ce dossier thématique vise à combler (partiellement !) ces vides et a pour ambition de contribuer aux recherches qui s'intéressent aux transformations du métabolisme urbain (Barles, 2015, 2017) en contexte de transition. Il s'inscrit dans les perspectives ouvertes par le champ de l'écologie territoriale (Buclet, 2015), dans celui de la political-industrial ecology (Newell, Cousins, 2015 ; Pincetl, Newell, 2017) et de l'écologie sociale de Vienne (Haberl et alii, 2016). S'il privilégie une entrée urbaine, il met l'accent sur l'inscription du métabolisme urbain dans un ensemble de flux qui dépasse largement les limites spatiales des villes. Comment peut-on en effet analyser les transformations du métabolisme urbain à court, moyen ou long terme ? Est-il possible de combiner des approches quantitatives et d'autres plus compréhensives, renvoyant tant aux sciences de la nature qu'aux sciences de l'homme et de la société ? Observe-t-on aujourd'hui les signes d'une transition socio-écologique, ou bien une consolidation du régime dominant par la survalorisation des signaux faibles (circuits 
courts, réseaux décentralisés, par exemple) ? Quels sont les effets des politiques de transition (économie circulaire, croissance verte, paquet énergie, etc.) sur le métabolisme urbain dans ses déclinaisons multiscalaires ? Quels sont les acteurs historiques et émergents du métabolisme urbain, les gagnants et les perdants de sa reconfiguration éventuelle ? En quoi la compréhension du métabolisme urbain permet-elle de révéler des inégalités et conflits spatiaux ? Comment les trajectoires de développement affectent-elles les trajectoires socioécologiques?

$\underline{3}$ Ces questions, dont on pourrait aisément allonger la liste, ouvrent un très vaste chantier qui est loin d'être terminé avec la publication de ce dossier. Celui-ci montre néanmoins, à nos yeux, l'importance d'une approche matérielle des sociétés urbaines en général, et en contexte d'urgence associée aux changements environnementaux tant locaux que planétaires en particulier.

\section{Différentes catégories de réseaux pour interroger le métabolisme urbain}

4Les contributions réunies dans le présent dossier apportent des connaissances nouvelles sur le métabolisme urbain et les processus socio-écologiques qui lui sont associés. Les textes se focalisent sur des études de cas variées pour analyser les différents registres des mutations métaboliques urbaines; ils traduisent les liens réciproques, et plus encore l'intrication existant entre métabolisme et réseaux sociotechniques.

5LLa première contribution est panoramique et s'attelle à une comparaison mondiale : en recueillant les données issues de 67 études de métabolisme urbain, Guilherme Iablonovski et Sabine Bognon interrogent les corrélations entre les indicateurs qui en résultent et des variables couramment associées à la performance écologique des territoires (par exemple la densité, l'étalement urbain, les modes de transports ou les espaces verts). Les auteurs montrent ainsi à la fois les régularités qui caractérisent le métabolisme urbain d'une ville à l'autre et les effets contradictoires du fonctionnement urbain, entre économies d'échelle et externalités négatives.

6Les trois contributions suivantes concernent la filière de construction en associant perspective métabolique et aménagement urbain. C'est une thématique essentielle dans ce dossier, car la consommation mondiale de matériaux de construction ne cesse d'augmenter emportant l'extraction minérale et l'accumulation de stocks à des niveaux impressionnants (Krausmann et alii, 2018) et impossibles à maintenir (Magalhães, 2019). Tout d'abord, Vincent Augiseau discute les contraintes liées à l'utilisation des déchets de la démolition et du renouvellement urbain dans la construction urbaine. À partir des résultats de l'analyse des flux de matière en Île-de-France démontrant que la masse de déchets est équivalente à celle des ressources primaires extraites, il en vient à se demander pourquoi ce flux de ressources secondaires est si peu réutilisé. C'est à partir de la littérature technique qu'il établit un diagnostic des contraintes expliquant ce constat, ce qui lui permet de déterminer des pistes d'actions à plusieurs échelles territoriales. L'article d'Agnès Bastin interroge les expérimentations de circuits réutilisant les déchets de construction, en miroir du précédent article. Elle mobilise le cadre théorique des transitions sociotechniques, au travers de l'approche multiniveaux (MLP), afin d'étudier le processus des changements de pratiques de ces acteurs. Cette analyse lui permet de comprendre le rôle de l'espace comme facteur de 
stabilité et, à l'opposé, de mutation des filières économiques de gestion des déchets inertes. Enfin, le travail de Mathieu Fernandez, Corinne Blanquart, Patrick Niérat et Éric

Verdeil investigue le lien entre consommation de matériaux de construction, émission de gaz à effet de serre et consommation d'espace à travers plusieurs scénarios appliqués à un vaste projet d'aménagement. Il montre qu'un scénario basé essentiellement sur la valorisation des déchets conduit à l'augmentation des distances de transports des matières (et donc des émissions), alors que le report modal (du routier au fluvial) contribue à l'extension urbaine. Ces trois articles sont donc très complémentaires en donnant à voir les paradoxes environnementaux, techniques et économiques de politiques urbaines a priori favorables à la transition dès lors qu'elles sont analysées avec un prisme métabolique. Il ne suffit donc pas de décréter que tel critère est durable, pour qu'il y ait dématérialisation des villes, planification urbaine plus économe en ressources (Perrotti, Stremke, 2018) ou désaccumulation des stocks (Wiedenhofer et alii, 2019).

7Le deuxième groupe d'articles concerne un autre grand flux du métabolisme urbain, celui de la biomasse et son corollaire les déchets organiques. Cette thématique aborde la transformation du métabolisme urbain autant au travers du rôle de l'agriculture urbaine ou périurbaine (McClintock 2010), que de celui de la récupération des rejets organiques urbains par différentes filières (Bahers, Giacchè, 2019 ; Dumain, Rocher, 2017). L'article de JeanDaniel Cesaro, Timothée Cantard, Marie-Lan Nguyen Leroy, Marie-Isabelle Peyre, Le Thi Thanh Huyen, et Guillaume Duteurtre évoque le rôle de paysans de la périphérie d'Hanoï qui collectent des biodéchets urbains pour nourrir leurs animaux d'élevage. Bien que la contribution de cette filière périurbaine soit inférieure à la mise en décharge des déchets organiques, il n'en reste pas moins qu'il s'agit d'une solution opérationnelle et singulière au gaspillage alimentaire. Cette recherche met aussi en relief l'enjeu de résistance de fermes paysannes à l'accaparement foncier de la métropolisation, ce qui offre une grille de lecture des tensions entre rural et urbain au cœur des relations métaboliques entre territoires. Par ailleurs, Élisabeth Lehec montre que le compostage en pied d'immeuble, en tant que dispositif décentralisé, est une technique de gestion des flux au même titre que le compostage industriel. À partir du cadre théorique de l'anthropologie des techniques d'André LeroiGourhan, ses résultats déconstruisent les oppositions entre réseaux centralisés et post-réseaux alternatifs, pour comprendre les différentes opérations matérielles et sociales de gestion du métabolisme urbain. Une autre approche est développée par Andrea Bortolotti, Stephan Kampelmann, Simon De Muynck, Anastasia Papangelou, Vanessa Zeller dans leur travail sur le métabolisme des biodéchets dans la ville-région de Bruxelles, qui met en avant et en débat une démarche interdisciplinaire pour développer et évaluer des scénarios de transition concernant leur gestion. Cet article offre ainsi un espace de réflexivité sur la mise en commun de l'expertise de quatre équipes de recherche dans le cadre de recherches sur le métabolisme urbain en présentant des moments de convergences et les limites de la collaboration. Ce groupe d'articles permet ainsi d'interroger les tensions inhérentes au traitement d'un flux sortant, pour reprendre les catégories métaboliques, les biodéchets. Ces tensions sont liées aux relations interterritoriales, aux techniques de gestion, et aux outils de représentations du métabolisme, apportant donc un éclairage sur l'hybridité des réseaux métaboliques (Coutard, Rutherford, 2009), réseaux qui ne sont pas réductibles à une filière ou un périmètre administratif.

$\underline{8}$ Cette hybridité peut également se caractériser par l'apport de l'économie informelle, qui est prépondérante dans la circulation des flux de sable, bois et déchets organiques à Ndzuwani aux Comores (Jean-Baptiste Bahers, Jeanne Perez, et Mathieu Durand) ou des déchets plastiques au Caire en Égypte (Pierre Desvaux). L'intérêt de ces deux articles est également 
le fait d'interroger le cadre théorique de l'urban political ecology (écologie politique urbaine). En effet, Pierre Desvaux avance une lecture des jeux de pouvoir qui conditionnent et structurent les flux métaboliques par la communauté cairote des zabbâlîn. Cela lui permet d'étudier finement un ensemble d'infrastructures techniques et sociales, qui connectent différents espaces par leurs pratiques. Jean-Baptiste Bahers, Jeanne Perez, et Mathieu Durand s'inspirent également de ce courant, au travers notamment des « inégalités métaboliques » de McFarlane (2013). Il en résulte la proposition de la notion de vulnérabilités métaboliques, qui embrasse les déterminants sociaux et spatiaux de la circulation des flux entre ce territoire insulaire et son hinterland. Les cas de ce métabolisme insulaire et de celui de la filière plastique cairote offrent des perspectives complémentaires des autres articles du dossier, en enrichissant les approches théoriques du métabolisme (Broto, Allen, Rapoport 2012 ; Pincetl, Bunje, Holmes, 2012) par l'informalité en tant que véritable contributrice du métabolisme et indispensable aux fonctionnements matériels et résilients des territoires (Guibrunet, Sanzana Calvet, Castán Broto, 2016).

9LLe dernier groupe d'articles, ceux de Jérémy Robert et d'Audrey Tanguy, aborde les enjeux d'approvisionnement - au sens de la gestion de la ressource -, qui sont absolument structurants dans les recherches sur le métabolisme urbain (Cousins et Newell 2015; Kim et Barles 2012). Jérémy Robert s'attache à comprendre le problème majeur de la disponibilité des ressources hydriques pour la capitale du Pérou, Lima, et comment les pouvoirs publics tentent d'y faire face. Leur gestion alternative est ainsi analysée au prisme des déterminants politiques de la transition métabolique. Il en résulte un processus paradoxal de mise à l'agenda politique d'une transition socio-écologique, et en même temps du retour aux projets de grandes infrastructures. Audrey Tanguy étudie, quant à elle, l'approvisionnement énergétique d'une ville portuaire (Saint-Nazaire). Elle met en évidence que plusieurs registres de compétitions sont structurants dans le métabolisme énergétique urbain entre technologies, entre installations décentralisées de production énergétique, et entre territoires d'approvisionnement et territoires de consommation. Cette dernière ligne de tension est particulièrement utile pour comprendre les transformations multiscalaires qui traversent le métabolisme urbain au travers de filières mondialisées et interconnectées de ressources (Crang et alii, 2013; Kissinger, Stossel 2019). En cela, il s'agit bien d'une critique de la dépendance du métabolisme urbain à ces réseaux et de l'économie politique de la circulation des matières.

10Pour finir, nous avons voulu réaliser un entretien avec un « chercheur-acteur » de ces mutations métaboliques en cours, afin d'éclairer le dossier d'une vision opérationnelle. Ainsi, Franck Scherrer est le directeur académique de l'Institut montréalais IEDDEC, et il nous apporte son regard sur les enjeux scientifiques de l'émergence de l'économie circulaire à l'échelle urbaine. Nous l'interrogeons donc sur la genèse de l'institut, mais aussi sur les controverses scientifiques qui sont associées à la notion d'économie circulaire. 
Bibliographie

Augiseau V., 2019, Utiliser les ressources secondaires de matériaux de construction : contraintes et pistes d'action pour des politiques territoriales, Flux, 2019/2-3 ( $\left.N^{\circ} 116-117\right)$, p. 26-41.

Bahers J.-B., Giacchè G., 2019, Towards a metabolic rift analysis: The case of urban agriculture and organic waste management in Rennes (France), Geoforum, 98 (janvier), p. 97-107.

DOI:10.1016/j.geoforum.2018.10.017

Bahers J.-B., Perez J., Durand M., 2019, Vulnérabilité métabolique et potentialités des milieux insulaires. Le cas de l'île de Ndzuwani (Anjouan), archipel des Comores, Flux, 2019/2-3 ( $\left.N^{\circ} 116-117\right)$, p. 128-146.

Barles S., 2017, Écologie territoriale et métabolisme urbain : quelques enjeux de la transition socioécologique, Revue d'Économie Régionale \& Urbaine, Décembre, p. 819-836. DOI:

10.3917/reru.175.0819

Barles S., 2015, The main characteristics of urban socio-ecological trajectories: Paris (France) from the 18th to the 20th century, Ecological Economics 118, p. 177-185. DOI:

10.1016/j.ecolecon.2015.07.027

Bastin A., 2019, Vers une gestion circulaire des matières inertes issues de la démolition et des travaux publics en région parisienne : une lecture croisant transition sociotechnique et approches territoriales, Flux, 2019/2-3 ( $\left.N^{\circ} 116-117\right)$, p. 42-57.

Bortolotti A., Kampelmann S., De Muynck S., Papangelou A., Zeller V., 2019, Conditions and concepts for interdisciplinary urban metabolism research-the case of an inter-project collaboration on biowaste, Flux, 2019/2-3 ( $\left.N^{\circ} 116-117\right)$, p. 112-127.

Broto V. C., Allen A., Rapoport E., 2012, Interdisciplinary perspectives on urban metabolism », Journal of Industrial Ecology, vol. 16 (6), p. 851-861.

Buclet N. (dir.), 2015, Essai d'écologie territoriale. L'exemple d'Aussois en Savoie, Paris : CNRS Editions.

Cesaro J.-D., Cantard T., Nguyen Leroy M.-L., Peyre M.-I., Le Thi Thanh Huyen, Duteurtre G., 2019, Les élevages-recycleurs de déchets alimentaires à Hanoï : un service informel en transition, Flux, 2019/23 (N¹16-117), p. 74-94.

Cousins J. J., Newell J. P., 201, A political-industrial ecology of water supply infrastructure for Los Angeles, Geoforum, 58, p. 38-50. DOI: 10.1016/j.geoforum.2014.10.011

Coutard O., Rutherford J., 2009, Les réseaux transformés par leurs marges : développement et ambivalence des techniques « décentralisées ", Flux, 2009/2 (n 76-77), p. 6-13. DOI :

10.3917/flux.076.0006 
Crang M., Hughes A., Gregson N., Norris L., Ahamed F., 2013, Rethinking Governance and Value in Commodity Chains through Global Recycling Networks, Transactions of the Institute of British Geographers, vol 38 (1), p. 12-24. DOI: 10.1111/j.1475-5661.2012.00515.x

Desvaux P., 2019, Pour une approche qualitative du métabolisme urbain. L'exemple des voies métaboliques des déchets plastiques au Caire (Égypte), Flux, 2019/2-3 ( $\left.N^{\circ} 116-117\right)$, p. 147-160.

Dumain A., Rocher L., 2017, Des pratiques citoyennes en régime industriel : les courts-circuits du compost, Flux, 2017/2 ( $N^{\circ} 108$ ), p. 22-35. DOI : 10.3917/flux1.108.0022

Fernandez M., Blanquart C., Niérat P., Verdeil É., 2019, Renouvellement urbain et optimisation du métabolisme : une équation complexe, Flux, 2019/2-3 ( $\left.N^{\circ} 116-117\right)$, p. 58-73.

Ferrao P., Fernández J. E., 2013, Sustainable Urban Metabolism, Cambridge, Massachusetts: 1st ed. MIT Press.

Guibrunet L., Sanzana Calvet M., Castan Broto V., 2016, Flows, system boundaries and the politics of urban metabolism: Waste management in Mexico City and Santiago de Chile, Geoforum, vol. 85. DOI: 10.1016/j.geoforum.2016.10.011

Haberl H., Fischer-Kowalski M., Krausmann F., Winiwarter V. (Eds.), 2016, Social Ecology, Cham: Springer International Publishing.

lablonovski G., Bognon S., 2019, Efficacité matérielle et performance écologique des territoires : Analyse croisée de 67 métabolismes, Flux, 2019/2-3 (N¹16-117), p. 6-25.

Kennedy C. A., Stewart I., Facchini A., Cersosimo I., Mele R., Chen B., Uda M., Kansal A., Chiu A., Kim K., Dubeux C., Rovere E. L. L., Cunha B., Pincetl S., Keirstead J., Barles S., Pusaka S., Gunawan J., Adegbile M., Nazariha M., Hoque S., Marcotullio P. J., Otharán F. G., Genena T., Ibrahim N., Farooqui R., Cervantes G., Sahin A. D., 2015, Energy and material flows of megacities, PNAS, 112, p. 5985-5990. DOI: $10.1073 /$ pnas.1504315112

Kim E., Barles S., 2012, The Energy Consumption of Paris and Its Supply Areas from the Eighteenth Century to the Present, Regional Environmental Change, vol. 12 (2), p. 295-310. DOI:

10.1007/s10113-011-0275-0

Kissinger M., Stossel Z., 2019, Towards an interspatial urban metabolism analysis in an interconnected world, Ecological Indicators, vol. 101, p. 1077-1085. DOI:

10.1016/j.ecolind.2018.11.022

Krausmann F., Lauk C., Haas W., Wiedenhofer D., 2018, From resource extraction to outflows of wastes and emissions: The socioeconomic metabolism of the global economy, 1900-2015, Global Environmental Change, vol. 52, p. 131-140. DOI: 10.1016/j.gloenvcha.2018.07.003

Lehec É., 2019, Vers un service composite de gestion du métabolisme urbain. Ce que compostage industriel et compostage en pied d'immeuble ont en partage, Flux, 2019/2-3 ( $\left.N^{\circ} 116-117\right)$, p. 95-111.

Magalhães N. 2019, Accumuler de la matière, laisser des traces, Terrestres, vol. 6, [En ligne] (consulté le 06 décembre 2019) Disponible à l'adresse : https://www.terrestres.org/2019/09/11/accumulerde-la-matiere-laisser-des-traces/ 
McClintock N., 2010, Why Farm the City? Theorizing Urban Agriculture through a Lens of Metabolic Rift, Cambridge Journal of Regions, Economy and Society, vol. 3 (2), p. 191-207. DOI:

10.1093/cjres/rsq005

McFarlane C., 2013, Metabolic inequalities in Mumbai, City, vol. 17, p. 498-503.

Newell J. P., Cousins J. J., 2015, The boundaries of urban metabolism: Towards a political-industrial ecology, Progress in Human Geography, vol. 39, p. 702-728. DOI: $10.1177 / 0309132514558442$

Perrotti D., Stremke S., 2018, Can Urban Metabolism Models Advance Green Infrastructure Planning? Insights from Ecosystem Services Research, Environment and Planning B: Urban Analytics and City Science. DOI: 10.1177/2399808318797131

Pincetl S., Bunje P., Holmes T., 2012, An expanded urban metabolism method: Toward a systems approach for assessing urban energy processes and causes, Landscape and Urban Planning, vol. 107 (3), p. 193-202. DOI: 10.1016/j.landurbplan.2012.06.006

Pincetl S., Newell J. P., 2017, Why data for a political-industrial ecology of cities?, Geoforum, vol 85. DOI: 10.1016/j.geoforum. 2017.03.002

Robert J., 2019, Trois innovations pour une transition ? L'émergence d'un tournant environnemental dans le métabolisme urbain de l'eau à Lima, Flux, 2019/2-3 ( $\left.N^{\circ} 116-117\right)$, p. 161-175.

Tanguy A., 2019, Le rôle des compétitions territoriales dans la transition énergétique, Flux, 2019/2-3 (N¹16-117), p. 176-191.

Wiedenhofer D., Fishman T., Lauk C., Haas W., Krausmann F., 2019, Integrating Material Stock Dynamics Into Economy-Wide Material Flow Accounting: Concepts, Modelling, and Global Application for 1900-2050, Ecological Economics, vol. 156, p. 121-33. DOI:

10.1016/j.ecolecon.2018.09.010

Weisz H., Steinberger J. K., 2010, Reducing energy and material flows in cities, Current Opinion in Environmental Sustainability, 2, p. 185-192. DOI: 10.1016/j.cosust.2010. 05.010

Zhang Y., Yang Z., Yu X., 2015, Urban Metabolism: A Review of Current Knowledge and Directions for Future Study, Environ. Sci. Technol., 49, p. 11247-11263. DOI: 10.1021/acs.est.5b03060 